\title{
Cysteine peptidases and their inhibitors in breast and genital cancer
}

\section{Anil Kumar Agrawal ${ }^{1}$, Godwin Bwire Ekonjo ${ }^{2}$, Elżbieta Teteryez ${ }^{2}$, Dorota Zyśko ${ }^{3}$, Zygmunt Grzebieniak $^{1}$, Magdalena Milan ${ }^{4}$, Grzegorz Marek ${ }^{1}$, Maciej Siewiński ${ }^{5,6}$}

\author{
${ }^{1}$ II Department of Surgery and Oncology, Wroclaw Medical University, Wroclaw, Poland \\ ${ }^{2}$ I Department of Obstetrics and Gynaecology, Wroclaw Medical University, Wroclaw, Poland \\ ${ }^{3}$ Teaching Department for Emergency Medical Services, Wroclaw Medical University, Wroclaw, Poland \\ ${ }^{4}$ Department of Clinical Procedures, Wroclaw Medical University, Wroclaw, Poland \\ ${ }^{5}$ Faculty of Public Healthy, Medical University of Wroclaw, Wroclaw, Poland \\ ${ }^{6}$ Beskid College of Skills in Żywiec, Poland
}

\begin{abstract}
Cysteine proteinases and their inhibitors probably play the main role in carcinogenesis and metastasis. The metastasis process need external proteolytic activities that pass several barriers which are membranous structures of the connective tissue which includes, the basement membrane of blood vessels. Activities of the proteinases are regulated by endogenous inhibitors and activators. The imbalance between cysteine proteinases and cystatins seems to be associated with an increase in metastatic potential in some tumors. It has also been reported that proteinase inhibitors, specific antibodies for these enzymes and inhibition of the urokinase receptor may prevent cancer cell invasion. Some proteinase inhibitor could serve as agents for cancer treatment.
\end{abstract}

Key words: cysteine peptidases, genital cancer, breast cancer, diagnosis, therapy.

\section{Introduction}

Cathepsin D, which is an aspartyl protease and directly activates other cathepsin, plays an important role in the tumour invasion and metastasis and is a subject of numerous reviews [1-3]. Cathepsin D is one of the main proteolytic enzymes contributing to the development of cancer [4].

In our review we would like to present the current state of knowledge regarding the meaning of the cysteine peptidases in breast and genital cancer prognosis.

\section{Cysteine proteinase inhibitors}

Cysteine proteinases are ultimately regulated by endogenous cysteine proteinase inhibitors. These inhibitors have been subdivided into three families, termed stefins, cystatins and kininogens. It is generally assumed that these inhibitors are involved in the

Correspondence: A.K. Agrawal, 2nd Department of General and Oncological Surgery, Borowska Str. 213, Medical University of Wrocław, 50-556 Wrocław, Poland; tel. (+48) 609289027, e-mai.dranilpreeti@gmail.com regulation of physiological and pathological processes caused by cysteine proteinases [5]. Cysteine proteinase inhibitors of all three families were found in ascitic fluid from patients with ovarian carcinoma. The highest apparent inhibition against cathepsin B was found in low molecular mass [6]. Kobayashi et al. demonstrated the presence of the anti-metastatic activity in the acid extract from human amniotic fluid. In previous it study was demonstrated that urinary trypsin inhibitor, which is one of the physiological trypsin inhibitors, isolated and purified from human urine, is able to inhibit tumor cell invasion [7]. Structurally more distant members of the cystatin superfamily have been reported, such as $\mathrm{p} 21$, the product of c-Ha-ras oncogene, which may be involved in earlier steps of tumor progression [8]. More recently, p41 a fragment from the invariant chain of the major histocompatibility complex (MHC) II, thyroglobulin types I domain and equinatoxin, an inhibitor from sea anemone, were discovered, all being cysteine proteinase inhibitors with no close structural homology to cystatins [9]. The recent discovery of two new cysteine proteinase inhibitors highlights the similarity between serine and 
proteinase inhibitors. The two new members of the serpin family (a family of serine proteinase inhibitors) appear to process potent inhibitory activity toward cysteine proteinases, and its ability to inhibit Interleukin 2-converting enzymes (ICE) and block the apoptotic process. This sepin was subsequently found to inhibit cathepsin L [10].

\section{The mechanism of interaction between cysteine proteinase inhibitors and cysteine proteinases}

The cystatins are reversible, tight-binding competitive inhibitors of cysteine proteinases. However, due to their extremely tight interactions with certain target enzymes, it has been difficult to verify, and dissociation equilibrium constants are difficult to determine accurately by equilibrium methods. A review of kinetic and structural data has enabled the authors to reconsider the definition of substrate binding sites in papainlike cysteine proteases. The location and definition of substrate binding sites beyond S3 and S2' are even more questionable. These results clearly indicate the differences in the specificity of interaction between the $\mathrm{N}$-terminal region of cystatin $\mathrm{C}$ and cathepsins $\mathrm{B}, \mathrm{H}, \mathrm{L}$ and $\mathrm{S}$, and that, although cystatin $\mathrm{C}$ has evolved to be a good inhibitor of all the mammalian cysteine proteinases, more specific inhibitors of the individual enzymes can be engineered $[11,12]$

Stopped-flow kinetics showed that the inhibition of the lysosomal cysteine proteinase, cathepsin B, by its endogenous inhibitor, cystatin $\mathrm{C}$, occurs by a two-step mechanism, in which an initial, weak interaction is followed by a conformational change. The initial interaction most likely involves binding of the N-terminal region of the inhibitor to the proteinase. The presence of this loop, which allows the enzyme to function as an exopeptidase, thus complicates the inhibition mechanism, rendering cathepsin $\mathrm{B}$ much less susceptible than other cysteine proteinases to inhibition by cystatins. The N-terminal region of human cystatin $\mathrm{C}$ has been shown to be of crucial importance for the interaction of the inhibitor with cysteine proteinases. These results show that bovine cystatin $\mathrm{C}$ has 118 residues, in contrast to 110-112 residues reported previously, and has an N-terminal region analogous to that of human cystatin $\mathrm{C}$. This region presumably is of similar importance for tight binding of target proteinases as a human proteases [13].

Inhibition of calpain by human kininogen domain 2 requires the correct conformation and combination of several contact sites, and this suggests that the N-terminus and the first hairpin loop play a major role in this ensemble. Remarkably, hybrid sc2-KD2 exhibited 5 and 150 times stronger inhibition of actinidin compared to native chicken cystatin and to proteolytically isolated from human kininogen domain 2, respectively. This indicates an important role of the first hairpin loop of cystatins in the interaction with actinidin. Along with the impaired inhibition of cathepsin L, papain, actinidin, cathepsin $\mathrm{B}$ and calpain by the hybrids sc1/3-KD2, sc2/3-KD2 and sc1/2/3-KD2, these results support the hypothesis that all three predicted contact regions of kininogen domain 2 contribute to binding in the active-site clefts of papain-like enzymes in a finely balanced manner [14]. The kininogens thus have the potential for binding two molecules of cysteine proteinases, the evidence regarding the binding stiochiometry of the intact molecules was conflicting. Recently, both intact LMK and HMK have been shown by several methods to bind two molecules of papain, cruzipain and cathepsins, $\mathrm{H}, \mathrm{L}$ and $\mathrm{S}$, thus clarifying the dilemma. Moreover, the two binding sites on HMK and LMK bind proteinase with different binding rates [15]. The inhibition of endopeptidases papain, cathepsins $\mathrm{S}$ and $\mathrm{L}$ and cruzipain by animal cystatins are extremely tight and ripped whereas the inhibition of exopeptidases cathepsins $\mathrm{B}$ and $\mathrm{H}$ is considerably weaker. The active site cleft of known endopeptidases is free to accommodate the inhibitors, whereas in the case of exopeptidases the active site cleft contains additional enzyme residues. In carboxydipeptidase cathepsin B its occluding loop partly occupies the active site cleft and needs to be displaced in order to accept a cystatin molecule [16].

\section{Breast cancer}

Cathepsin D was first of the lysosomal enzymes proved to be of prognostic significance for survival of breast cancer patients [17]. Podgorski and Sloane [18] reported that a group of breast cancer patients with low cathepsin B levels experienced poor survival. In a recent study of matched pairs of breast tumors and control breast tissues, increased activity and protein levels of cathepsin B proved to be of a better prognostic indicator value than those of cathepsin L, while cathepsin D increase did not show this [19]. In a lymph node negative population, cathepsin $\mathrm{B}$ protein and activity were better prognostic indicator for diseasefree survival than in the total patient population. On the other hand the expression of cathepsin B in gastric carcinoma is associated with lymph node metastasis but not with postoperative survival [20], the negative cathepsin D staining was related with one year survival in advanced squamous cell carcinoma [21] and cathep$\sin \mathrm{K}$ and cystatin $\mathrm{C}$ were not predictors of the survival in patients with advanced non-small-cell lung cancer during chemotherapy [22]. In Harcbeck and al. study a large increase in cathepsins B, L enzymatic activity was measured relative to their protein concentrations, indicating highly enhanced activation of cathepsins in 
tumors [19]. The latter may be due either to the enhanced activation of their precursors and/or to the downregulation of the endogenous inhibitors. Indeed, a significant decrease of stefin A level in some, but not all tumor was observed. Immunohistochemical analysis of stefin A in breast tumors revealed an opposite relation to survival compared with the studies on activity and protein level. Munster and Norton [23] found that risks of death was significantly higher in patients with positive staining of stefin A in tumors. The increase in risk was also significant in lymph node negative patients. Furthermore, co-expression of stefin A and p53 were indicative for high-risk patients. In the recent study it was found that 57 patient out of 1,500 with primary breast carcinoma, both cathepsin B and cathepsin L levels were found to be effective in predicting the rate of relapse and the length of survival after removal of the primary tumor. The levels, measured in tumor tissue cytosols, were positively correlated with the number of positive lymph nodes and negatively with the level of the steroid-hormones receptors. No significant relationship was found between cathep$\sin \mathrm{B}$ and L levels and age, menopausal status of the patients and size of the primary tumor. The levels of cathepsin B and L positively correlated with each other and the relapse-free and overall survival in univariate analysis. In multivariate regression analysis, corrected for age, menopausal status, tumor size, the number of invaded lymph nodes and steroid-hormone receptor status, cathepsin B and L were found to be significant independent factors for prediction of the rates of relapse and death. It was also found that the estrogeninduced degradation of estrogen-receptor (ER) protein could be prevented by pretreatment with peptide aldehyde inhibitors of proteasome protease whereas inhibitors of calpain and lysosomal proteases were ineffective. Inhibition of proteasome activity, maintained ER protein at a level equivalent to control cells not stimulated with estrogens but increased estrogensbinding activity by 1.75 -fold. The comparative prognostic value of Cathepsin D and urokinase plasminogen activator was detected by immunohistochemistry, in primary breast carcinoma [24]. In node-negative breast cancer, $70 \%$ of the patients were treated by surgery alone and thus there is a necessity of systemic adjuvant treatment. Histomorphological and tumor biological prognostic factors may be employed to assess the patient's risk profile with regard to disease recurrence and death. Cysteine protease cathepsin L, serine protease $\mathrm{UPA}$, and the protease inhibitor PAI-1 were determined by ELISA in extracts of primary tumors of 103 node-negative breast cancer patients and values assessed by univariate and multivariate analysis in comparison with traditional prognostic factors (tumor size, steroid hormone receptor status, grading, vessel invasion, menopausal status). Combined deter- mination of PAI-1 and cathepsin L tumor levels lowrisk node-negative breast cancer patients may be identified. These patients most probably will not benefit from systemic adjuvant therapy [25]. Proteinases are attractive targets for drug development in therapeutic application. The use of novel inhibitors in clinical practice is dependent not just on their specificity and effectiveness, but mainly on the knowledge of their precise role in the mechanisms of the proteolytic system in the development of malignant disease [26]. The lysosomal cysteine protease inhibitor 9-fluorenylmethyloxycarbonyl-tyrosylalanyl-diazomethane was found to inhibit growth of the breast cancer cell lines SK-Br-3 and MCF-7. The conjugated inhibitor was delivered and targeted to cathepsin $\mathrm{B}$ and an unidentified protein of $\mathrm{Mr} 39,000$ in the SK-Br-3 cells. The results in this report show that targeting of cysteine protease inhibitors can selectively control tumor cell growth and that targeted cysteine protease inhibitors could prove valuable in the development of novel anticancer immunotherapies. Bowman-Birk inhibitor (BBI) is a soybean-derived anticarcinogenic protease inhibitor previously shown to potentate cisplatininduced cytoxicity in human lung and ovarian cancer cells. To further assess the potential of BBI as a sensitizing agent for cancer radiotherapy and chemotherapy, they evaluated the effects of BBI and a soybean concentrate enriched in BBI known as BBI concentrate (BBIC) on clonogenic survival and radiation- or cisplatin-induced cell killing in human breast carcinoma cells, head and neck, carcinoma cells, human cervical carcinoma cells, and nontumorigenic human epithelial cells. These results suggest that BBIC could be a useful agent for the potentiation of radiation- and cisplatin-mediated cancer treatment without significant adverse effects on surrounding normal tissues [27]. Urinary trypsin inhibitor (UTI) which is one of the physiological trypsin inhibitors, isolated and purified from human urine is able to inhibit lung metastasis of muse Lewis lung carcinoma (3LL) cells in experimental and spontaneous metastasis model. The combination of an anti-metastasis (UTI) with an anti-cancer drug, etoposide showed a significant reduction in the formation of lung metastasis, and might provide a therapeutical basis for anti-metastatic therapy $[28,29]$.

The present results indicate that release of lysosomal cysteine proteinase from the lysosomal compartments is of little or no importance in the photochemical inactivation of NHIK 3025 cells when TPPS4 is used as photosensitizer. All human biological fluids and tissues investigated showed cysteine proteinase inhibiting activity, but it is not known whether the above-mentioned inhibitors account for major part of this activity; neither it is not known, nor it is known whether their relative concentrations vary between different biological sources, and if they have the capacity 
to play an important physiological and pathophysiological roles as cysteine proteinase inhibitors [30], reported that human amniotic fluid has anti-metastasis factor of molecular mass about 60-70 kDa identified as trypsin proteinase inhibitor. Recent findings on the role of cathepsins $\mathrm{B}, \mathrm{H}$, and $\mathrm{L}$ and their endogenous inhibitors in breast carcinomas as biological prognostic markers in human cancers were reviewed. We observed that cat B and procat B activities were elevated 45-fold and 24-fold respectively in tumor patients with ovarian carcinomas as compared with controls tissue. An imbalance between cysteine proteinase and endogenous cysteine proteinase inhibitors in ovarian and breast carcinomas was possibly due to impaired inhibition of proteinases. Alternatively, less effective inhibition by the endogenous inhibitors in tumors may be responsible for the activation of cysteine cathepsins. Secreted procath B is able to bind extracellular matrix components such as laminin or collegians, and eventually it is activated extracellulary by cat D. Premzl et al. [31] studied the effects of E-64 (CPI) on metastasis formation in mice with mammary and ovarian tumors. The data suggests that cat B, L, and D seem to be involved in early steps of the metastasis process rather than in the haematogenous spread of tumor cells, and other pharmacological activities which may account for the discrepant effects of E-64 on experimental and spontaneous metastasis cannot be ruled out. The ability to block invasive tumor cells in vitro with cysteine proteinase inhibitors supports a role for active cysteine proteinases in the invasion of some tumor cells. Bervar et al. [32] have recently shown that cystatin A was associated with negative BcI-2 staining, but there was no statistically significant association between axially lymph node status and p53 immunostaining in breast cancer, showing that a novel Immunohistochemical staining pattern involving CPI cystatin A in breast tumors was associated with poor histological differentiation. Proteinase inhibitors became targets for drug development in anti-tumor therapy. To our knowledge, this is the first report on in vivo application of photodynamic therapy together with cysteine proteinase inhibitor [33].

\section{Clinical relevance of cysteine proteinases in genital cancer}

Human ovarian cancer is characterised by its tendency to extend over the peritoneal surface of the abdominal cavity, resulting into a wide-spread disease. At its terminal stages, multiple metastatic foci appear in distant organs, possibly with the involvement of proteolytic enzymes. Ovarian cancer cell lines express detectable and reproducible levels of surface urokinase-type plasminogen activator and cathepsin B [34]. A latent, high molecular mass form of cathepsin B, presumably procathepsin $\mathrm{B}$, has been shown to accumulate in malignant ascitic fluids, among others from patients with ovarian cancer $[35,36]$. Cathepsin B activity, determined in serum was suggested as a factor for estimating the extent and spread of uterine cervical carcinoma [37]. The activity of this enzyme increased both in serum and in the tumor with the progression of the disease and was dependent on the clinical stage of cervical carcinoma. Ovarian carcinomas secrete single-chain urinary-type plasminogen activator (scuPA) and expression of UPA is up-regulated relative to normal ovarian epithelium, leading to an enhanced proteolytic capacity that may facilitate invasion. Both NTF and scuPA induced a dose-dependent increase in proliferation, with maximal stimulation obtained at 10-20 nM. Furthermore, blocking the interaction of endogenous UPA with UPAR using anti-NTF antibodies significantly inhibited proliferation. Together these datas indicate that, in addition to enhancing the invasive activity of ovarian carcinoma cells via increased pericellular proteolysis, uPA also acts as a mitogen for ovarian carcinoma cells, suggesting a biochemical mechanism whereby uPA may contribute to ovarian carcinoma progression by modulating both cell invasion and proliferation [37]. Furthermore, in the spontaneous metastasis model, the hybrid protein inhibited the formation of lung and/or lymphatic metastasis by human ovarian carcinoma and choriocarcinoma cells. The hybrid protein was much more effective than uPA-(1-134)-peptide, UTI-(78-136)-peptide, or UTI. That concludes that this approach extends the possibility of applying recombinant protein for therapeutic use in inhibition of human tumor cell metastasis [38-40].

\section{Conclusion}

The function of cysteine peptidases and their inhibitors in genital and breast cancer is not yet well known, but many researches suggest that they play a main role in carcinogenesis and metastasis. Some investigators point out that cysteine proteinases can be used as an independent factor for prediction of death rates in patients with breast cancer. Its also important to clear out that this can be used as an observation tool during and after therapy. The levels of cysteine peptidases and their inhibitors can be helpful in identifying patients with lymph node negative breast cancer, who will not benefit from systemic adjuvant therapy. Proteinase inhibitors may also be useful in inhibition of human tumor cell metastasis especially in genital tumors.

\section{References}

[ 1] Minarowska A, Gacko M, Karwowska A, Minarowski Ł. Human cathepsin D. Folia Histochem Cytobiol. 2008;46(1): 23-38.

[2] Gacko M, Minarowska A, Karwowska A, Minarowski Ł. Cathepsin D inhibitors. Folia Histochem Cytobiol. 2007; 45(4):291-313.

[3] Minarowska A, Minarowski L, Karwowska A, Gacko M. Regulatory role of cathepsin D in apoptosis. Folia Histochem Cytobiol. 2007;45(3):159-63. 
[ 4] Chabowski A, Sulkowska M, Sulkowski M, Famulski W, Skrzydlewska E, Kisielewski W. Immunohistochemical evaluation of cathepsin D expression in colorectal cancer. Folia Histochem Cytobiol. 2001;39(2):153-4.

[5] Rawlings ND, Tolle DP, Barrett A.J. Evolutionary families of peptidase inhibitors. Biochem J. 2004;378:705-716.

[ 6] Nishikawa H, Ozaki H, Nakanishi T et al. The role of cathep$\sin \mathrm{B}$ and cystatin $\mathrm{C}$ in the mechanisms of invasion by ovarian cancer. Gynecol Oncol. 2004;92:881-886.

[7] Kobayashi H, Shinohara H, Gotoh J et al. Anti-metastatic therapy by urinary trypsin inhibitor in combination with an anti-cancer agent. British J Cancer 1995;72:1131-1137.

[ 8] Kuang SQ, Liao L, Zhang H et al. AIB1/SRC-3 deficiency affects insulin-like growth factor I signaling pathway and suppresses v-Ha-ras-induced breast cancer initiation and progression in mice. Cancer Res. 2004;64:1875-1885.

[ 9] Lenarčič B, Turk V. Thyroglobulin type - 1domains in equitation inhibit both papain-like cysteine proteinases and cathepsin D. J Biol Chem. 1999;274:563-566.

[10] Gerl R, Vaux D. Apoptosis in the development and treatment of cancer. Carcinogenesis. 2005;26:263-270.

[11] Pavlova A, Bjork I. Grafting of features of cystatins C or B into the $\mathrm{N}$-terminal region or second binding loop of cystatin A (stefin A) substantially enhances inhibition of cysteine proteinases. Biochemistry. 2003;42:11326-11333.

[12] Turk D, Guncar G, Podobnik M, Turk B. Revised definition of substrate binding sites of papain-like cysteine proteases. Biol Chem. 1998;379:137-147.

[13] Turk D, Guncar G, Podobnik M, Turk B. Revised definition of substrate binding sites of papain-like cysteine proteases. Biol Chem. 1998;379:137-147.

[14] Auerwald EA, Nogler DK, Gross S. Hybrids of chicken cystatin with human kininogen domain 2 sequences exhibit novel inhibition of calpain, improved inhibition of actinidin and impaired inhibition of papain, cathepsin L and cathepsin B. Eur J Biochem. 1996;235:534-542.

[15] Barros NM, Tersariol IL, Oliva ML. High molecular weight kininogen as substrate for cathepsin B. Biol Chem. 2004;385: 551-555.

[16] Muehlenweg B, Assflag-Machleidt I, Parado SG. A novel type of bifunctional inhibitor directed against proteolytic activity and receptor/ligand interaction. Cystatin with a urokinase receptor binding site. J Biol Chem. 2000;275:3356233566.

[17] Avisar E, McParland E, Dicostanzo D, Axelrod D. Prognostic factors in node-negative male breast cancer. Clin Breast Cancer 2006;7:331-335.

[18] Podgorski I, Sloane BF. Cathepsin B and its role(s) in cancer progression. Biochem Soc Symp. 2003;70:263-276.

[19] Harbeck N, Alt U, Berger U et al. Prognostic impact of proteolytic factors (urokinase-type plasminogen activator, plasminogen activator inhibitor 1 , and cathepsins $\mathrm{B}, \mathrm{D}$, and $\mathrm{L}$ ) in primary breast cancer reflects effects of adjuvant systemic therapy. Clin Cancer Res. 2001;7:2757-2764.

[20] Czyżewska J, Guzińska-Ustymowicz K, Kemona A, Bandurski R. The expression of matrix metalloproteinase 9 and cathepsin B in gastric carcinoma is associated with lymph node metastasis, but not with postoperative survival. Folia Histochem Cytobiol. 2008;46(1):57-64.

[21] Szumilo J, Burdan F, Zinkiewicz K. et al. Expression of syndecan-1 and cathepsins $\mathrm{D}$ and $\mathrm{K}$ in advanced esophageal squamous cell carcinoma. Folia Histochem Cytobiol. 2009; 47(4):571-8

[22] Naumnik W, Niklińska W, Ossolińska M, Chyczewska E. Serum cathepsin $\mathrm{K}$ and cystatin $\mathrm{C}$ concentration in patients with advanced non-small-cell lung cancer during chemotherapy. Folia Histochem Cytobiol. 2009;47(2):20713.
[23] Munster PN, Norton L. Predictive factor for the response to adjuvant therapy with emphasis in breast cancer. Breast Cancer Res. 2001;3:361-364.

[24] Esteva FJ, Hortobagvi GN. Prognostic molecular markers in early breast cancer. Breast Cancer Res. 2004;6:109-118.

[25] Herszenyi L, Plebani M, Carraro P et al. The role of cysteine and serine proteases in colorectal carcinoma. Cancer. 1999; 86:1135-1142.

[26] Chen YW, Huang SC, Lin-Shiau SY, Lin JK. Bowman-Birk inhibitor abates proteasome function and suppresses the proliferation of MCF7 breast cancer cells through accumulation of MAP kinase phosphatase-1. Carcinogenesis 2005;26:12961306.

[27] Kobayashi H, Shinohara H, Gotoh J et al. Anti-metastatic therapy by urinary trypsin inhibitor in combination with an anti-cancer agent. British J Cancer. 1995;72:1131-1137.

[28] Roberts R. Lysosomal cysteine proteases: structure, function and inhibition of cathepsins. Drug News Perspect. 2005; 18:605-614.

[29] Kobayashi H, Suzuki M, Sun GW et al. Suppression of urokinase-type plasminogen activator expression from human ovarian cancer cells by urinary trypsin inhibitor. Biochim Biophys Acta. 2000;1481:310-316.

[30] Kobayashi H, Suzuki M, Hirashima Y, Terao T. The protease inhibitor bikunin, a novel anti-metastatic agent. Biol Chem. 2003;384:749-754.

[31] Premzl A, Turk V, Kos J. Intracellular proteolytic activity of cathepsin B is associated with capillary-like tube formation by endothelial cells in vitro. $J$ Cell Biochem. 2006;97:12301240.

[32] Bervar A, Zajc I, Sever N et al. Invasiveness of transformed human breast epithelial cell lines is related to cathepsin B and inhibited by cysteine proteinase inhibitors. Biol Chem. 2003;384:447-455.

[33] Saleh Y, Ziółkowski P, Siewiński M et al. The combined treatment of transplantable solid mammary carcinona in wistar rats by use photodynamic therapy and cysteine proteinase inhibbitors. In vivo. 2005;15:351-357.

[34] Kobayashi H, Ohi H, Sugimura M et al. Inhibition of in vitro ovarian cancer cell invasion by modulation of urokinase-type plasminogen activator and cathepsin B. Cancer Res. 1993;52: 3610-3614.

[35] Keppler D, Abrahamson M, Sordat B. Secretion of cathepsin B and tumour invasion. Biochem Soc Trans. 1994;22:43-49.

[36] Siewiński M, Saleh Y, Popiela A et al. Expression of high molecular weight cysteine proteinase inhibitor in ovarian cancer tissues: Regulation of cathepsin B expression by placental CPI. Biol Chem. 2003;384:1103-1107.

[37] Daneri-Navarro A, Del Toro-Arreola S, Sanchez-Hernandez $\mathrm{PE}$ et al. Immunosuppressive activity of proteases in cervical carcinoma. Gynecol Oncol. 2005;98:111-117.

[38] Henic E, Sixt M, Hansson S et al. EGF-stimulated migration in ovarian cancer cells is associated with decreased internalization, increased surface expression, and increased shedding of the urokinase plasminogen activator receptor. Gynecol Oncol. 2006;101:28-39.

[39] Kobayashi H, Sugino D, She MY et al. A bifunctional hybrid molecule of the amino-terminal fragment of urokinase and domain II of bikinis efficiently inhibits tumor cell invasion and metastasis. Eur J Biochem. 1998;253:817-826.

[40] Kobayashi H, Hirashima Y, Sun GW et al. Identification and characterization of a Kunitz-type protease inhibitor in ascites fluid from patients with ovarian carcinoma. Int $J$ Cancer 2000;87:44-54.

Submitted: 12 May, 2010 Accepted after reviews: 11 August, 2010 